\title{
Application of the Elliptically Polarized Radio Frequency Fields in Spin-3/2 Nuclear Quadrupole Resonance Spectroscopy
}

\author{
Anna Sklyar ${ }^{\mathrm{a}}$, Nikolay Sinyavsky ${ }^{\mathrm{b}}$, Michal Ostafin $^{\mathrm{a}}$, and Boleslaw Nogaj ${ }^{\mathrm{a}}$ \\ a Department of Physics Adam Mickiewicz University, Umultowska 85, 61-614, Poznan, Poland \\ b Baltic State Academy, Molodiozhnaya str. 6, 236029, Kaliningrad, Russia \\ Reprint requests to N. S.; E-mail: sinyavsky_physics@ bga.gazinter.net
}

Z. Naturforsch. 64a, 618-624 (2009); received June 23, 2008 / revised June 21, 2009

\begin{abstract}
A possibility to excite the spin-3/2 quadrupolar nuclei in sites with a non-zero asymmetry parameter of the electric field gradient (EFG) tensor by means of an elliptically polarized radio frequency (RF) magnetic fields is discussed. Closed analytical formulas for the intensities of nuclear quadrupole resonance (NQR) nutation spectra and nutation frequencies of powder samples were obtained. Characteristic singularities in the nutation spectra were determined which allow the measurement of the asymmetry parameter $\eta$. It was found that in the general case of $\eta \neq 0$ the excitation of the nuclear spins in $+m$ and $-m$ states by using the circularly polarized RF fields is not fully selective.
\end{abstract}

Key words: Nuclear Quadrupole Resonance; Elliptically Polarized RF Field; Electric Field Gradient Tensor; Asymmetry Parameter.

\section{Introduction}

For spin $I=3 / 2$ and other half-integer spin nuclei the spins in states $\pm m$ have an equal nuclear quadrupole interaction energy but undergo a precession in opposite directions in the EFG frame. In a normal NQR experiment a linearly polarized RF field is used so that these two oppositely precessing components of nuclear magnetization are excited simultaneously [1]. This is possible because a linearly polarized RF field can be decomposed into two circularly polarized components rotating in opposite directions and the two circular components of nuclear magnetization induced that way result in a net linearly polarized component oscillating along the direction of the linearly polarized RF field.

The application of circularly instead of linearly polarized RF fields in NQR was reported for the first time in [1] and it allowed there the excitation of only one half of the total nuclear spin system, i.e. the subsystem with $+m$ or $-m$. This selective excitation of the $I=3 / 2$ nuclear spin system also enabled the authors of [1] to consider the possible quantum transition between the degenerate states of the nuclear spin system separately and thus a precise determination of the contribution of nuclear quadrupole relaxation into an NQR signal. Their study, however, was confined to a single-crystal sample and an axial symmetry of the
EFG tensor. Of much more interest obviously would be exploring a general case of a polycrystalline sample with non-axial symmetry of the EFG tensor. This would include the analysis of line shapes and singularities in the nutation NQR spectra obtained with an elliptically polarized RF field applied to the sample. Using for this purpose the elliptically instead of the circularly polarized RF fields seems to be useful because the elliptically polarized RF field can be decomposed into two circularly polarized components rotating in the opposite directions but with different amplitudes. So, unlike for circular polarization, the nuclear spins in $\pm m$ states should be excited by elliptically polarized fields with a noticeable difference.

Circularly polarized RF magnetic field has also been used in [2] for spin $I=1$ nuclei and allowed to increase the $\mathrm{S} / \mathrm{N}$ ratio of ${ }^{14} \mathrm{~N}-\mathrm{NQR}$ signals by the factor of $\sqrt{2}$, a significant result when the application of NQR spectroscopy for explosive detection is considered.

Nutation NQR spectroscopy using linearly polarized RF magnetic fields has been well developed in papers [3-9] and is now a routine method used for studying specific aspects of electronic structure of molecular crystals. The goal of this paper is a theoretical study of the possibility to use the elliptically polarized RF fields for recording the nutation NQR spectra of spin $I=3 / 2$ nuclei in powder samples and the determina- 
tion of asymmetry parameter $\eta$ from the positions of nutation spectra singularities.

\section{Theory}

To calculate the free induction decay (FID) signals observed after applying an RF pulse of elliptically polarized magnetic field a method similar to that mentioned in [10] can be used. However, unlike to [10], where the coefficients $C_{n}$ were used in interaction representation, in order to obtain the final results for $\left\langle I_{x, y, z}\right\rangle$ one can define $C_{n}$ in a laboratory frame. The response of the nuclear spin system with $I=3 / 2$, experiencing the nuclear quadrupole interaction in zero magnetic field after a single RF pulse, can be studied by using the non-stationary theory of perturbations. We shall assume that in the time interval $0 \leq t \leq t_{w}$ the nuclear spin system is in a thermodynamic equilibrium with the lattice until the RF pulse is turned on. Because the elapsed time of the experiment is significantly smaller than the spin-lattice relaxation time $\mathrm{T}_{1}$ we can assume that the spin system is virtually isolated from the lattice and consequently all the nuclear quadrupole relaxation effects will be neglected.

Let the axis of the RF sample coil, which produces a pulse of the RF field $B_{1}$, be directed at arbitrary angles $(\theta, \varphi)$ with respect to the principal axes of the EFG tensor. A second RF coil producing a pulse of the RF field $B_{1}^{\prime}$ is perpendicular with respect to the first one (angles $\theta^{\prime}, \varphi^{\prime}$ ) and produces RF oscillations which are phase shifted by $90^{\circ}$ with respect to the first one. We shall describe the response of the nuclear spin system to a short RF pulse of a duration $t_{w}$ and this duration is much smaller than the time of reaching a new thermal equilibrium in a spin system experiencing the presence of the RF fields $B_{1}$ and $B_{1}^{\prime}$, i. e. $t_{w} \ll T_{2} \rho$.

To calculate the NQR signal after the RF pulse with an elliptically polarized field one must solve the time dependent (non-stationary) Schroedinger equation in the time interval $0 \leq t \leq t_{w}$. To obtain the expectation values of the quantities $\left\langle I_{x, y, z}\right\rangle$ at an arbitrary time $t \geq t_{w}$, we use a wave function of the form $[1,3]$

$$
\Psi=\sum_{i} C_{i}\left(t_{w}\right) \cdot \varphi_{i} \mathrm{e}^{-\mathrm{i} \omega_{i}\left(t-t_{w}\right)},
$$

where $\varphi_{i}$ are the orthogonal eigenfunctions of the quadrupole Hamiltonian $H_{Q}$ and $\omega_{i}=\frac{E_{i}}{\hbar}$ are the energy levels of the nuclear quadrupole interaction. The quadrupole Hamiltonian matrix for $I=3 / 2$ has the form:

$$
\begin{gathered}
H_{Q}=\frac{e Q q_{z z}}{4}\left[\begin{array}{cccc}
1 & \frac{\eta}{\sqrt{3}} & 0 & 0 \\
\frac{\eta}{\sqrt{3}} & -1 & 0 & \frac{\eta}{\sqrt{3}} \\
0 & 0 & -1 & 0 \\
0 & 0 & \frac{\eta}{\sqrt{3}} & 1
\end{array}\right] \Rightarrow \\
U^{-1} H_{Q} U=\frac{e Q q_{z z}}{4} \rho\left[\begin{array}{cccc}
1 & 0 & 0 & 0 \\
0 & -1 & 0 & 0 \\
0 & 0 & -1 & 0 \\
0 & 0 & 0 & 1
\end{array}\right],
\end{gathered}
$$

where $U$ is the unitary matrix of transformation to the eigenvalue representation

$$
U=\left[\begin{array}{cccc}
\sqrt{\frac{\rho+1}{2 \rho}} & -\sqrt{\frac{\rho-1}{2 \rho}} & 0 & 0 \\
\sqrt{\frac{\rho-1}{2 \rho}} & \sqrt{\frac{\rho+1}{2 \rho}} & 0 & 0 \\
0 & 0 & \sqrt{\frac{\rho+1}{2 \rho}} & \sqrt{\frac{\rho-1}{2 \rho}} \\
0 & 0 & -\sqrt{\frac{\rho-1}{2 \rho}} & \sqrt{\frac{\rho+1}{2 \rho}}
\end{array}\right],
$$

where $\rho=\sqrt{1+\frac{\eta^{2}}{3}}$. The frequency of transitions between the two double degenerate energy levels is $\omega_{0}=$ $e Q q_{z z} \rho / 2 \hbar$. Coefficients $C_{k}\left(t_{w}\right)$ can be determined by solving the non-stationary Schoedinger equation

$$
\mathrm{i} \hbar \dot{\psi}=\left[H_{Q}+H(t)\right] \psi
$$

over the time interval $0 \leq t \leq t_{w}$. Utilizing the orthogonality properties of the eigenfunctions $\varphi_{i}(i=1,2,3,4)$, we get from (4) a system of equations to be solved for $C_{n}$ :

$$
\mathrm{i} \hbar \frac{\mathrm{d} C_{k}}{\mathrm{~d} t}=\sum_{n} V_{k n} C_{n} \cdot \mathrm{e}^{-\mathrm{i} \omega_{k n} t},
$$

where $\omega_{k n}=\frac{E_{k}-E_{n}}{\hbar}$ is a transition frequency between two energy levels. The interaction Hamiltonian for the RF field $B_{1}$, having elliptical polarization and being shifted by $90^{\circ}$ with respect to the field $B_{1}^{\prime}$, produced by the second coil can be written as:

$$
\vec{H}=\vec{H}_{1}+\vec{H}_{1}^{\prime}=-\gamma \hbar\left(\vec{B}_{1} \cos \omega_{0} t+\vec{B}_{1}^{\prime} \sin \omega_{0} t\right) \vec{I}(6)
$$

and $\omega=\omega_{0}$ is the spectrometer frequency set exactly on resonance. The $H_{1}$ matrix has the form $H_{1}=$ $-\gamma \hbar B_{1} \cos \omega_{0} t \cdot V_{k n}$, with the elements $V_{k n}$ given by 


$$
\left[\begin{array}{cccc}
\frac{\rho+2}{2 \rho} \cos \theta & -\frac{\eta}{\sqrt{3} \rho} \cos \theta & \frac{\sin \theta}{2 \sqrt{3} \rho}\left(3 \mathrm{e}^{\mathrm{i} \varphi}+\eta \mathrm{e}^{-\mathrm{i} \varphi}\right) & \frac{\sin \theta}{2 \rho}\left[\eta \mathrm{e}^{\mathrm{i} \varphi}+\mathrm{e}^{-\mathrm{i} \varphi}(\rho-1)\right] \\
-\frac{\eta}{\sqrt{3} \rho} \cos \theta & \frac{\rho-2}{2 \rho} \cos \theta & -\frac{\sin \theta}{2 \rho}\left[\eta \mathrm{e}^{\mathrm{i} \varphi}-\mathrm{e}^{-\mathrm{i} \varphi}(\rho+1)\right] & \frac{\sin \theta}{2 \sqrt{3} \rho}\left(3 \mathrm{e}^{\mathrm{i} \varphi}+\eta \mathrm{e}^{-\mathrm{i} \varphi}\right) \\
\frac{\sin \theta}{2 \sqrt{3} \rho}\left(3 \mathrm{e}^{-\mathrm{i} \varphi}+\eta \mathrm{e}^{\mathrm{i} \varphi}\right) & -\frac{\sin \theta}{2 \rho}\left[\eta \mathrm{e}^{-\mathrm{i} \varphi}-\mathrm{e}^{\mathrm{i} \varphi}(\rho+1)\right] & -\frac{\rho-2}{2 \rho} \cos \theta & \frac{\eta}{\sqrt{3} \rho} \cos \theta \\
\frac{\sin \theta}{2 \rho}\left[\eta \mathrm{e}^{-\mathrm{i} \varphi}+\mathrm{e}^{\mathrm{i} \varphi}(\rho-1)\right] & \frac{\sin \theta}{2 \sqrt{3} \rho}\left(3 \mathrm{e}^{-\mathrm{i} \varphi}+\eta \mathrm{e}^{\mathrm{i} \varphi}\right) & \frac{\eta}{\sqrt{3} \rho} \cos \theta & -\frac{\rho+2}{2 \rho} \cos \theta
\end{array}\right]
$$

Passing for the rotating coordinate system we can rewrite $H_{1}$ as $H_{1}=-\gamma \hbar B_{1} \cos \omega_{0} t V_{k n} \mathrm{e}^{\mathrm{i} \omega_{k n} t}$, where $\omega_{k n}=$ $\left(E_{k}-E_{n}\right) / \hbar$. Considering the two perpendicular RF fields and retaining the time independent terms only we obtain:

$$
H=H_{1}+H_{1}^{\prime}=\left[\begin{array}{cccc}
0 & -A_{z}+\mathrm{i} A_{z}^{\prime} & A_{x}-\mathrm{i} A_{x}^{\prime}+A_{y}^{\prime}+\mathrm{i} A_{y} & 0 \\
-A_{z}-\mathrm{i} A_{z}^{\prime} & 0 & 0 & A_{x}+\mathrm{i} A_{x}^{\prime}-A_{y}^{\prime}+\mathrm{i} A_{y} \\
A_{x}+\mathrm{i} A_{x}^{\prime}+A_{y}^{\prime}-\mathrm{i} A_{y} & 0 & 0 & A_{z}+\mathrm{i} A_{z}^{\prime} \\
0 & A_{x}-\mathrm{i} A_{x}^{\prime}-A_{y}^{\prime}-\mathrm{i} A_{y} & A_{z}-\mathrm{i} A_{z}^{\prime} & 0
\end{array}\right] .
$$

Here the vector $\vec{B}_{1}$ with coordinates $B_{1 x}=$ $B_{1} \sin \theta \cos \varphi, B_{1 y}=B_{1} \sin \theta \sin \varphi$, and $B_{1 z}=B_{1} \cos \theta$ was replaced by the vector $\vec{A}$ with coordinates $A_{x, y}=\frac{\gamma(\eta \pm 3)}{4 \sqrt{3} \rho} B_{1 x, y}$ and $A_{z}=\frac{\gamma \eta}{2 \sqrt{3} \rho} B_{1 z}$. We used a vector $\vec{A}^{\prime}$ similarly, instead of the vector $\vec{B}_{1}^{\prime}$ with coordinates $B_{1 x}^{\prime}=B_{1}^{\prime} \sin \theta^{\prime} \cos \varphi^{\prime}, B_{1 y}^{\prime}=B_{1}^{\prime} \sin \theta^{\prime} \sin \varphi^{\prime}$, and $B_{1 z}^{\prime}=B_{1}^{\prime} \cos \theta^{\prime}$.

Then the system of differential equations (5) can be written as:

$$
\begin{aligned}
& \dot{C}_{1}=-\mathrm{i}\left(H_{12} C_{2}+H_{13} C_{3}\right), \\
& \dot{C}_{2}=-\mathrm{i}\left(H_{21} C_{1}+H_{24} C_{4}\right), \\
& \dot{C}_{3}=-\mathrm{i}\left(H_{31} C_{1}+H_{34} C_{4}\right), \\
& \dot{C}_{4}=-\mathrm{i}\left(H_{42} C_{2}+H_{43} C_{3}\right),
\end{aligned}
$$

where $H_{i j}$ are the elements of the matrix (8).

We shall seek for the solutions of system (9) taking the initial conditions $C_{1}(0)=C_{4}(0)=0, C_{2}(0)=$ $\mathrm{e}^{\mathrm{i} \Phi} / \sqrt{2}$, and $C_{3}(0)=1 / \sqrt{2}$ into consideration because the nuclear spin system is in thermal equilibrium until the RF pulse is turned on. Here $\Phi$ is a hypothetical random phase. Thus, when the RF pulse is turned on the $C_{m}$ coefficients will take the form:

$$
\begin{aligned}
& C_{1}\left(t_{w}\right)=-\frac{\mathrm{i}}{2 \sqrt{2}}\left[\left(1+d_{z}\right)\left(G_{x}+\mathrm{i} G_{y}-\mathrm{e}^{\mathrm{i} \Phi} G_{z}\right)\right. \\
& \left.+\left(d_{x}+\mathrm{i} d_{y}\right)\left(-\mathrm{e}^{\mathrm{i} \Phi} G_{x}+\mathrm{ie}^{\mathrm{i} \Phi} G_{y}-G_{z}\right)\right] \frac{\sin \alpha_{+} t_{w}}{\alpha_{+}} \\
& -\frac{\mathrm{i}}{2 \sqrt{2}}\left[\left(1-d_{z}\right)\left(G_{x}+\mathrm{i} G_{y}-\mathrm{e}^{\mathrm{i} \Phi} G_{z}\right)\right. \\
& \left.-\left(d_{x}+\mathrm{i} d_{y}\right)\left(-\mathrm{e}^{\mathrm{i} \Phi} G_{x}+\mathrm{ie}^{\mathrm{i} \Phi} G_{y}-G_{z}\right)\right] \frac{\sin \alpha_{-} t_{w}}{\alpha_{-}},
\end{aligned}
$$

$$
\begin{aligned}
& C_{2}\left(t_{w}\right)=-\frac{1}{2 \sqrt{2}}\left[-d_{x}-\mathrm{i} d_{y}+\mathrm{e}^{\mathrm{i} \Phi}\left(d_{z}-1\right)\right] \cos \alpha_{+} t_{w} \\
& +\frac{1}{2 \sqrt{2}}\left[-d_{x}-\mathrm{i} d_{y}+\mathrm{e}^{\mathrm{i} \Phi}\left(d_{z}+1\right)\right] \cos \alpha_{-} t_{w} \\
& C_{3}\left(t_{w}\right)=\frac{1}{2 \sqrt{2}}\left[d_{z}+1+\mathrm{e}^{\mathrm{i} \Phi}\left(d_{x}-\mathrm{i} d_{y}\right)\right] \cos \alpha_{+} t_{w} \\
& -\frac{1}{2 \sqrt{2}}\left[d_{z}-1+\mathrm{e}^{\mathrm{i} \Phi}\left(d_{x}-\mathrm{i} d_{y}\right)\right] \cos \alpha_{-} t_{w} \\
& C_{4}\left(t_{w}\right)=-\frac{\mathrm{i}}{2 \sqrt{2}}\left[-\left(d_{x}-\mathrm{i} d_{y}\right)\left(G_{x}+\mathrm{i} G_{y}-\mathrm{e}^{\mathrm{i} \Phi} G_{z}\right)\right. \\
& \left.-\left(d_{z}-1\right)\left(\mathrm{e}^{\mathrm{i} \Phi} G_{x}-\mathrm{ie}^{\mathrm{i} \Phi} G_{y}+G_{z}\right)\right] \frac{\sin \alpha_{+} t_{w}}{\alpha_{+}} \\
& -\frac{\mathrm{i}}{2 \sqrt{2}}\left[\left(d_{x}-\mathrm{i} d_{y}\right)\left(G_{x}+\mathrm{i} G_{y}-\mathrm{e}^{\mathrm{i} \Phi} G_{z}\right)\right. \\
& \left.+\left(d_{z}+1\right)\left(\mathrm{e}^{\mathrm{i} \Phi} G_{x}-\mathrm{ie}^{\mathrm{i} \Phi} G_{y}+G_{z}\right)\right] \frac{\sin \alpha_{-} t_{w}}{\alpha_{-}}
\end{aligned}
$$

where $G_{x}=A_{x}-\mathrm{i} A_{x}^{\prime}, G_{y}=A_{y}-\mathrm{i} A_{y}^{\prime}, G_{z}=A_{z}-\mathrm{i} A_{z}^{\prime}$, and

$$
\alpha_{+,-}=\sqrt{|\vec{A}|^{2}+\left|\vec{A}^{\prime}\right|^{2} \pm 2\left|\vec{A} \times \overrightarrow{A^{\prime}}\right|}
$$

At any moment of time $t$ after the pulse $t_{w}$ is off the coefficients $C_{m}$ will have the following form:

$$
C_{m}(t)=C_{m}\left(t_{w}\right) \cdot \mathrm{e}^{-\mathrm{i} \omega_{m}\left(t-t_{w}\right)},
$$

where $\omega_{m}=E_{m} / \hbar$. The expectation values of the components of the nuclear magnetization after the RF pulse is off can be determined from the equation

$$
\left\langle I_{q}\right\rangle=\sum_{i, j} C_{i}^{*} C_{j}\left\langle i\left|I_{q}\right| j\right\rangle \cdot \mathrm{e}^{-\mathrm{i}\left(\omega_{i}-\omega_{j}\right)\left(t-t_{w}\right)},
$$

where $q=x, y, z,\left\langle i\left|I_{q}\right| j\right\rangle$ are matrix elements of the $\hat{q}$ operators in $\hat{H}_{Q}$ representation and $t$ is the current 
time measured starting from the RF pulse end. After simple transformations of (13) we obtain:

$$
\begin{aligned}
\left\langle I_{x}\right\rangle= & \frac{3+\eta}{2 \sqrt{3} \rho}\left[\left(C_{1}^{*} C_{3}+C_{4}^{*} C_{2}\right) \mathrm{e}^{-\mathrm{i} \omega_{0}\left(t-t_{w}\right)}\right. \\
& \left.+\left(C_{2}^{*} C_{4}+C_{3}^{*} C_{1}\right) \mathrm{e}^{\mathrm{i} \omega_{0}\left(t-t_{w}\right)}\right] \\
\left\langle I_{y}\right\rangle= & \mathrm{i} \frac{3-\eta}{2 \sqrt{3} \rho}\left[\left(C_{1}^{*} C_{3}-C_{4}^{*} C_{2}\right) \mathrm{e}^{-\mathrm{i} \omega_{0}\left(t-t_{w}\right)}\right. \\
& \left.+\left(C_{2}^{*} C_{4}-C_{3}^{*} C_{1}\right) \mathrm{e}^{\mathrm{i} \omega_{0}\left(t-t_{w}\right)}\right] \\
\left\langle I_{z}\right\rangle= & \frac{\eta}{\sqrt{3} \rho}\left[\left(C_{4}^{*} C_{3}-C_{1}^{*} C_{2}\right) \mathrm{e}^{-\mathrm{i} \omega_{0}\left(t-t_{w}\right)}\right. \\
& \left.+\left(C_{3}^{*} C_{4}-C_{2}^{*} C_{1}\right) \mathrm{e}^{\mathrm{i} \omega_{0}\left(t-t_{w}\right)}\right]
\end{aligned}
$$

Substituting (12) into (14) and averaging the result over the random phase $\Phi$ we obtain the mean values of the operators $I_{x, y, z}$ :

$$
\begin{aligned}
\left\langle I_{x}\right\rangle= & -\frac{3+\eta}{4 \sqrt{3} \rho}\left[\left(A_{x}^{\prime}+F_{x}\right) \frac{\sin 2 \alpha_{+} t_{w}}{\alpha_{+}}\right. \\
& \left.+\left(A_{x}^{\prime}-F_{x}\right) \frac{\sin 2 \alpha_{-} t_{w}}{\alpha_{-}}\right] \cos \omega_{0}\left(t-t_{w}\right) \\
& +\frac{3+\eta}{4 \sqrt{3} \rho}\left[\left(A_{x}-F_{x}^{\prime}\right) \frac{\sin 2 \alpha_{+} t_{w}}{\alpha_{+}}\right. \\
& \left.+\left(A_{x}+F_{x}^{\prime}\right) \frac{\sin 2 \alpha_{-} t_{w}}{\alpha_{-}}\right] \sin \omega_{0}\left(t-t_{w}\right), \\
\left\langle I_{z}\right\rangle= & -\frac{\eta}{2 \sqrt{3} \rho}\left[\left(A_{z}^{\prime}+F_{z}\right) \frac{\sin 2 \alpha_{+} t_{w}}{\alpha_{+}}\right. \\
& \left.+\left(A_{z}^{\prime}-F_{z}\right) \frac{\sin 2 \alpha_{-} t_{w}}{\alpha_{-}}\right] \cos \omega_{0}\left(t-t_{w}\right) \\
& +\frac{\eta}{2 \sqrt{3} \rho}\left[\left(A_{z}-F_{z}^{\prime}\right) \frac{\sin 2 \alpha_{+} t_{w}}{\alpha_{+}}\right. \\
& \left.+\left(A_{z}+F_{z}^{\prime}\right) \frac{\sin 2 \alpha_{-} t_{w}}{\alpha_{-}}\right] \sin \omega_{0}\left(t-t_{w}\right), \\
\left\langle I_{y}\right\rangle= & -\frac{3-\eta}{4 \sqrt{3} \rho}\left[\left(A_{y}^{\prime}+F_{y}\right) \frac{\sin 2 \alpha_{+} t_{w}}{\alpha_{+}}\right. \\
& \left.+\left(A_{y}^{\prime}-F_{y}\right) \frac{\sin 2 \alpha_{-} t_{w}}{\alpha_{-}}\right] \cos \omega_{0}\left(t-t_{w}\right) \\
& +\frac{3-\eta}{4 \sqrt{3} \rho}\left[\left(A_{y}-F_{y}^{\prime}\right) \frac{\sin 2 \alpha_{+} t_{w}}{\alpha_{+}}\right. \\
& \left.+\left(A_{y}+F_{y}^{\prime}\right) \frac{\sin 2 \alpha_{-} t_{w}}{\alpha_{-}}\right] \sin \omega_{0}\left(t-t_{w}\right),
\end{aligned}
$$

where $\vec{F}=\vec{d} \times \vec{A}, \vec{F}^{\prime}=\vec{d} \times \vec{A}^{\prime}, \vec{d}=\frac{\vec{D}}{|\vec{D}|}$, and $\vec{D}=\vec{A} \times \vec{A}^{\prime}$. Also, we assumed that $\vec{d} \vec{G}=0$.

The NQR signal induced in the first RF coil is given by the quantity

$G(t)=\left\langle I_{x}\right\rangle \sin \theta \cos \varphi+\left\langle I_{y}\right\rangle \sin \theta \sin \varphi+\left\langle I_{z}\right\rangle \cos \theta$.
Thus, taking into consideration that $\vec{A} \vec{F}=\vec{A}^{\prime} \vec{F}^{\prime}=0$, we obtain

$$
\begin{aligned}
& G(t)=-\frac{1}{\gamma B_{1}} \vec{A} \vec{A}^{\prime}\left[\frac{\sin 2 \alpha_{+} t_{w}}{\alpha_{+}}\right. \\
& \left.+\frac{\sin 2 \alpha_{-} t_{w}}{\alpha_{-}}\right] \cos \omega_{0}\left(t-t_{w}\right) \\
& +\frac{1}{\gamma B_{1}}\left[\vec{A}\left(\vec{A}-\vec{F}^{\prime}\right) \frac{\sin 2 \alpha_{+} t_{w}}{\alpha_{+}}\right. \\
& \left.+\vec{A}\left(\vec{A}+\vec{F}^{\prime}\right) \frac{\sin 2 \alpha_{-} t_{w}}{\alpha_{-}}\right] \sin \omega_{0}\left(t-t_{w}\right) .
\end{aligned}
$$

Similarly, for the signal induced in the second RF coil $G^{\prime}(t)=\left\langle I_{x}\right\rangle \sin \theta^{\prime} \cos \varphi^{\prime}+\left\langle I_{y}\right\rangle \sin \theta^{\prime} \sin \varphi^{\prime}+\left\langle I_{z}\right\rangle \cos \theta^{\prime}$, we obtain

$$
\begin{aligned}
G^{\prime}(t)=- & \frac{1}{\gamma B_{1}^{\prime}}\left[\vec{A}^{\prime}\left(\overrightarrow{A^{\prime}}+\vec{F}\right) \frac{\sin 2 \alpha_{+} t_{w}}{\alpha_{+}}\right. \\
& \left.+\vec{A}^{\prime}\left(\vec{A}^{\prime}-\vec{F}\right) \frac{\sin 2 \alpha_{-} t_{w}}{\alpha_{-}}\right] \cos \omega_{0}\left(t-t_{w}\right) \\
+ & \frac{1}{\gamma B_{1}^{\prime}} \vec{A}^{\prime} \vec{A}\left[\frac{\sin 2 \alpha_{+} t_{w}}{\alpha_{+}}\right. \\
& \left.+\frac{\sin 2 \alpha_{-} t_{w}}{\alpha_{-}}\right] \sin \omega_{0}\left(t-t_{w}\right) .
\end{aligned}
$$

\section{Results and Discussion}

As can be seen from (17) and (18), excitation of the nuclear spins by an RF field with elliptical polarization results in two nutation frequencies $\omega_{n+}=2 \alpha_{+}$ and $\omega_{n-}=2 \alpha_{-}$unlike using the linearly polarized RF field. In the particular case, when a single RF coil and a linearly polarized field are used to excite the NQR signal, we get from (17)

$$
G(t)=\frac{2 \alpha}{\gamma B_{1}} \sin 2 \alpha t_{w} \sin \omega_{0}\left(t-t_{w}\right),
$$

because in these conditions $\vec{A}^{\prime}=\vec{D}=\vec{F}=\vec{F}^{\prime}=0$ and $\alpha_{+}=\alpha_{-}=\alpha$, where

$$
\begin{aligned}
2 \alpha=\frac{\gamma B_{1}}{2 \sqrt{3} \rho} & {\left[4 \eta^{2} \cos ^{2} \theta\right.} \\
& \left.+\sin ^{2} \theta\left(9+\eta^{2}+6 \eta \cos 2 \varphi\right)\right]^{1 / 2} .
\end{aligned}
$$

The equations for intensities (19) and for nutation frequencies (20) appear to be consistent with those known earlier [10].

As the analysis of (15) shows, in the general case of arbitrary angles of orientations of the two mutually perpendicular coils with respect to the principal 
a)
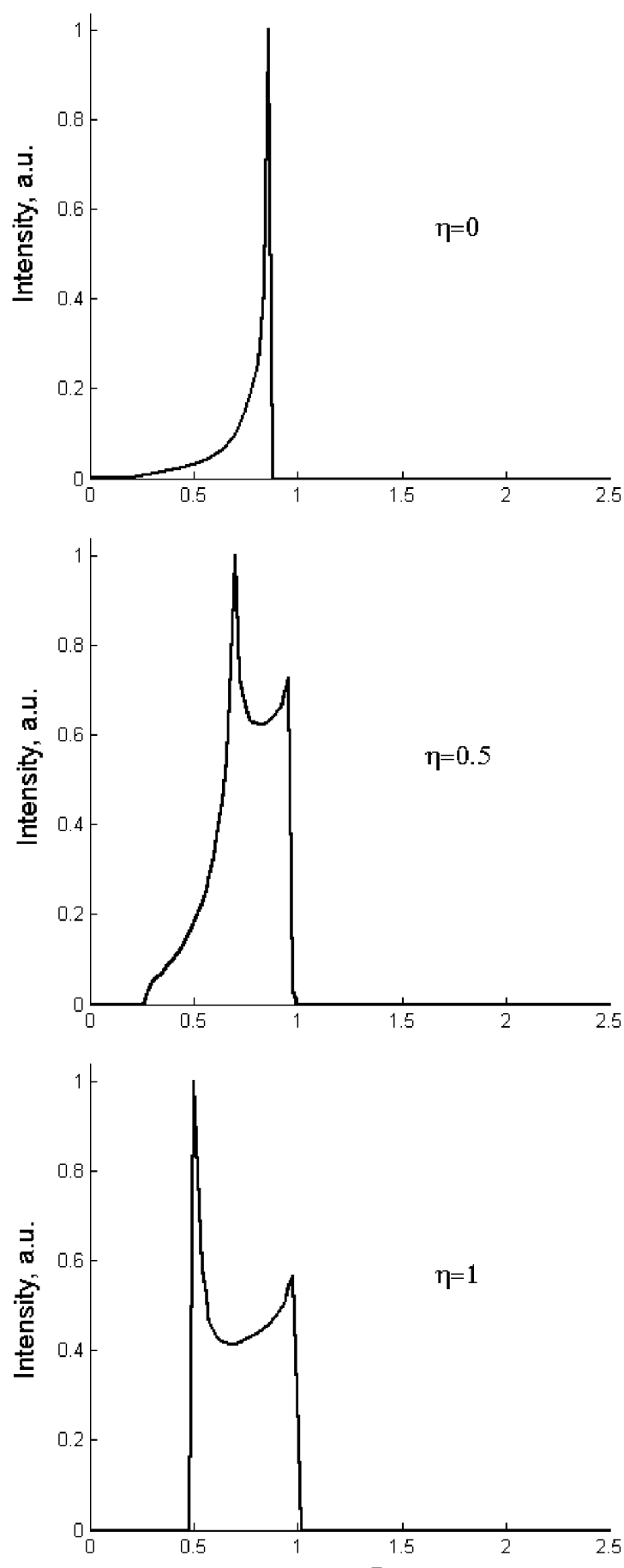

$\omega_{n} / \mathrm{B}_{1}$ b)
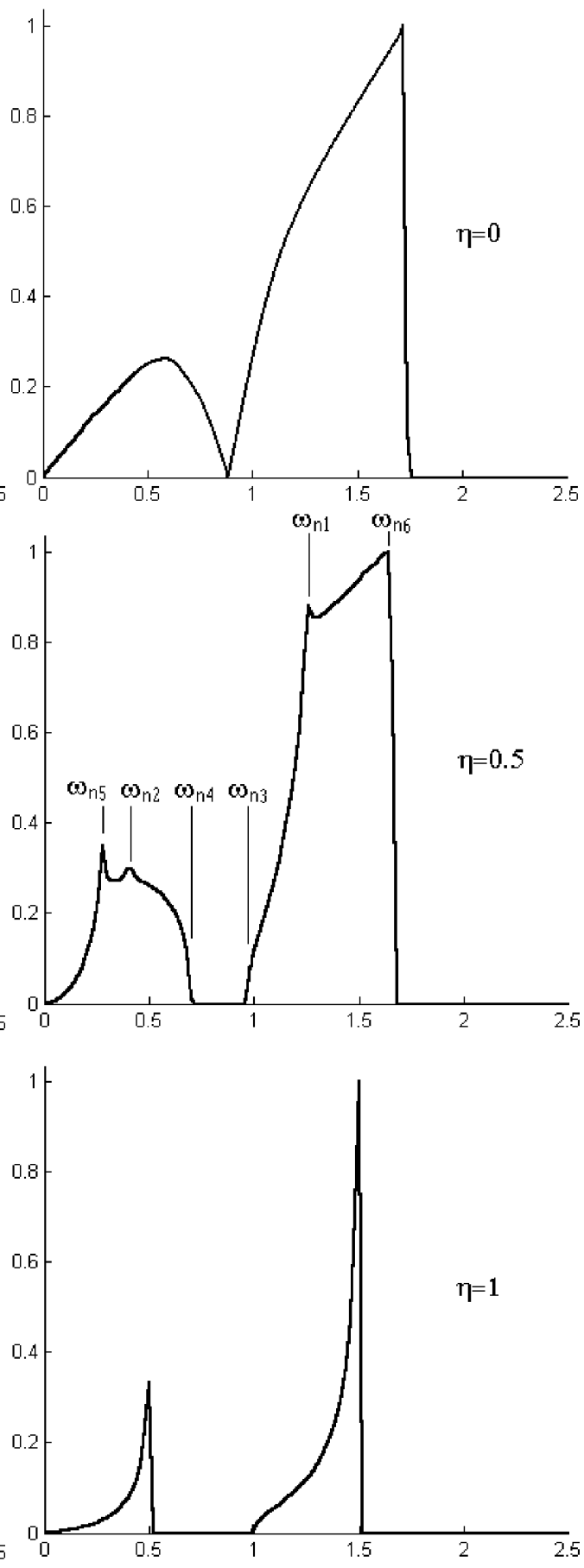

$\omega_{n} / y B_{1}$

Fig. 1. Simulated nutation NQR spectra of powders for excitation of the nuclear quadrupole transitions by the linearly (a) and circularly (b) polarized RF magnetic fields. 
axes of the EFG tensor the nuclear magnetization will have spatially elliptical polarization. However, the coefficient of eccentricity of this ellipse will depend on the aforementioned angles and the asymmetry parameter $\eta$. This implies that the nuclear spins in states $+m$ and $-m$ will be somewhat differently excited and the excitation selectivity of both states will be different. Even for the simple case of a monocrystalline sample, where the RF coil is directed along the $x, y$ principal axes of the EFG tensor $\left(B_{1}=B_{1}^{\prime}\right)$ and the absence of axial symmetry of the latter, the nuclear magnetization rotating in the principal $x y$-plane will be elliptically polarized and not circularly like in [1], where $\eta=0$ was assumed.

For a polycrystalline sample (17) and (18) should be averaged over all possible angles $\theta, \varphi, \theta^{\prime}$, and $\varphi^{\prime}$ and for the two perpendicular transmitting coils the following condition should hold: $\sin \theta \sin \theta^{\prime} \cos \varphi \cos \varphi^{\prime}+$ $\sin \theta \sin \theta^{\prime} \sin \varphi \sin \varphi^{\prime}+\cos \theta \cos \varphi^{\prime}=0$.

Theoretical nutation NQR spectra obtained by the method of partial summation of spectral intensities for the case of linearly and circularly polarized RF fields and for different values of the asymmetry parameter are shown in Figure 1a and b, respectively.

In the general case of circularly polarized field $\left(B_{1}=B_{1}^{\prime}\right)$ and polycrystalline samples the nutation spectra show two broad but non overlapping spectra with characteristic frequency singularities given by:

$$
\begin{aligned}
& \omega_{n 1,2}=\gamma B_{1} \frac{\sqrt{3}(1 \pm \eta)}{2 \rho}, \quad \omega_{n 3,4}=\gamma B_{1} \frac{3 \pm \eta}{2 \sqrt{3} \rho}, \\
& \omega_{n 5}=\gamma B_{1} \frac{\eta}{\sqrt{3} \rho}, \quad \omega_{n 6}=\gamma B_{1} \frac{\sqrt{3}}{\rho} .
\end{aligned}
$$

The dependencies of these singularities on the asymmetry parameter of the EFG tensor are shown in Figure 2. The positions of the singularities $\omega_{n 1}-\omega_{n 6}$ are also marked on one of the spectra in Figure 1b.

Unlike for the nutation NQR spectra of powders obtained with linearly polarized RF fields and consisting of a single line with characteristic shape, when the circularly polarized RF field is applied the nutation powder pattern consists of two nutation lines of different intensity and shape. The low frequency line results from a selective excitation of the nuclear spin states $+m$ and $-m$. The high frequency line results from the excitation mode for which the precessing magnetization is directed along the axis of rotation of the RF field.

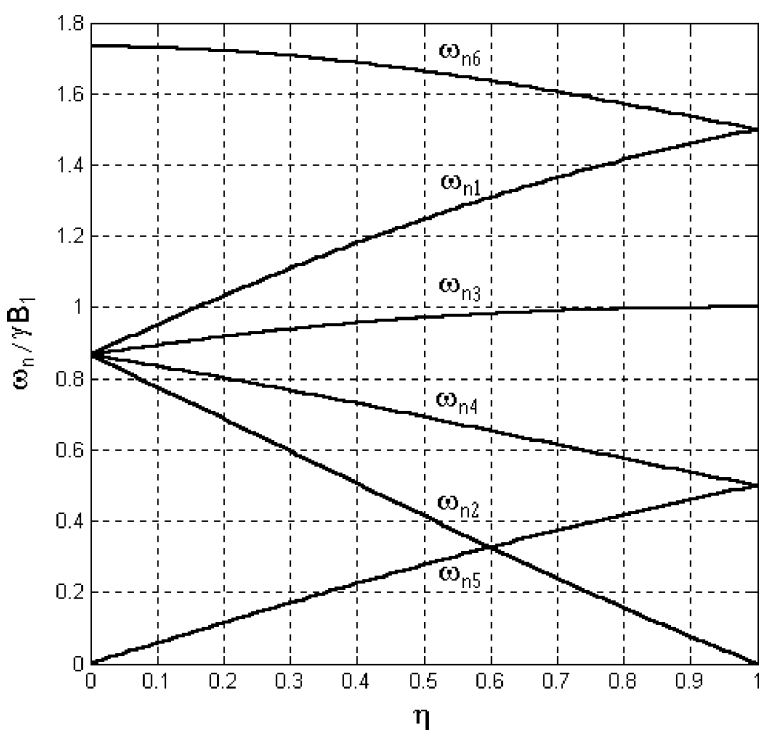

Fig. 2. The dependence of the positions of frequency singularities in an NQR nutation spectra of powders on asymmetry parameter $\eta$ for excitation of NQR transitions by circularly polarized RF magnetic fields.

The dependence of the positions of frequency singularities of nutation spectra on $\eta$ allows its experimental determination. For spin $I=3 / 2$ nuclei, as it is well known, it is not possible to extract $\eta$ form the measurement of pure quadrupole frequency alone because the latter depends on both $\eta$ and the nuclear quadrupole coupling constant $e Q q_{z z}$ :

$$
\omega_{0}=\frac{e Q q_{z z}}{2 \hbar} \sqrt{1+\frac{\eta^{2}}{3}} .
$$

The NQR signal induced in the second RF coil (18) for a polycrystalline sample gives the nutation spectrum similar to that shown in Figure 1b. This signal appears only to be shifted by $90^{\circ}$ with respect to a similar nutation spectrum obtained from the signal given by (17). Addition or subtraction of NQR signal induced in both RF coils allows increasing the $\mathrm{S} / \mathrm{N}$ ratio by $\sqrt{2}$ and thus refining the singularity positions.

\section{Conclusions}

In this work, for the first time as we believe, the analytical formulas for intensities of the NQR signals of spin $I=3 / 2$ nuclei were obtained for a general case of powder samples excited with RF resonant magnetic fields which are elliptically polarized. We have shown that in a general case of arbitrary directions of prin- 
cipal axes of an EFG tensor and a non-zero asymmetry parameter $\eta$ of the latter the freely precessing nuclear magnetization is also elliptically polarized. The degree of deviation of this polarization from circularity depends on the orientations of the principal axes of the EFG tensor and on $\eta$. In a general case for every single crystallite in a sample there are two nutation frequencies which depend on the relative orientation of the EFG principal axes with respect to the two exciting RF coils and on $\eta$. Unlike in the single-crystal case, for powders it is not possible to perform fully selective excitation of the two nuclear spin subsystems in states characterized by quantum numbers $\pm m$, respectively, by applying a circularly polarized RF field and regardless the direction of rotation of the latter.

Nutation NQR powder patterns for $I=3 / 2 \mathrm{nu}$ clei are theoretically calculated and computer sim-

[1] M. J. Weber and E. L. Hahn, Phys. Rev. 120, 365 (1960).

[2] J. B. Miller, B. H. Suits, and A. N. Garroway, J. Magn. Reson. 151, 228 (2001).

[3] G. S. Harbison and A. Slokenbergs, Z. Naturforsch. 45a, 575 (1990).

[4] N. Sinyavsky, M. Ostafin, and M. Mackowiak, Z. Naturforsch. 51a, 363 (1996).

[5] N. Sinjavsky, M. Ostafin, and M. Mackowiak, Appl. Magn. Reson. 15, 215 (1998). ulated for the first time. These calculations indicated, that the nutation patterns consist of two nonoverlapping nutation spectra with characteristic singularities and functional dependence of the positions of these singularities on $\eta$ was found. Based on this dependence a new method could be devised to determine $\eta$ from nutation spectra in powder samples by means of RF magnetic fields with circular polarization. This approach appears to be a good alternative to other methods, which have been used so far for this purpose.

\section{Acknowledgement}

One of us (N.S.) thanks the foundation "Kasa im. Jozefa Mianowskiego - Fundacja Popierania Nauki" (Poland) for financial support.

[6] N. Sinyavsky, M. Mackowiak, and N. Velikite, Z. Naturforsch. 54a, 153 (1999).

[7] N. Sinyavsky and M. Mackowiak, Molecular Physics Reports 37, 48 (2003).

[8] N. Sinyavsky and M. Mackowiak, Z. Naturforsch. 59a, 228 (2004).

[9] I. Korneva, N. Sinyavsky, M. Ostafin, B. Nogaj, and M. Mackowiak, Solid State NMR 31, 119 (2007).

[10] J.C. Pratt, P. Raghunathan, and C.A. McDowell, J. Magn. Reson. 20, 313 (1975). 